\title{
Neurology meets Psychiatry
}

\section{Liebe Kolleginnen, liebe Kollegen,}

seit unserem letzten Schwerpunktheft Grenzgebiete der Neurologie und Psychiatrie im Januar 2006 wurde ich oft auf die Problematik der Facharztausbildung beider Fächer angesprochen. Dabei zeigte sich, dass diese Problematik nach wie vor sehr emotional geführt wird und sich wahrscheinlich auch keine sachliche Lösung finden wird. Auffällig war, dass die Sichtweisen der niedergelassenen Kollegen und der Klinikärzte sehr unterschiedlich sind. In diesen Gesprächen forderten Klinikärzte in der Neurologie mehrheitlich, das Pflichtjahr Psychiatrie abzuschaffen. Psychiater in der Klinik hatten eine ambivalente Einstellung und niedergelassene Neurologen befürworteten mehrheitlich dieses „Pflichtjahr“.

Ich bin zwar auch der Meinung, dass die augenblickliche Situation nicht allen Anforderungen gerecht wird, aber eine wirklich bessere Lösung als die aktuell bestehende sehe ich nicht. Meines Erachtens ist der Einblick, den wir in das andere Fach bekommen, sogar zu gering. Wahrscheinlich gilt dies nicht für unsere Kollegen auf den Intensivstationen, aber für alle Neurologen, die in der Praxis und in weniger spezialisierten Abteilungen arbeiten. Wahr- scheinlich müsste die Ausbildung stärker auf die Bedürfnisse ausgerichtet sein und sollte nicht als bloße Pflicht gesehen werden. (Übrigens habe auch ich viel von den Kollegen aus der Psychiatrie gelernt, die bei uns in der Neurologie ihr Pflichtjahr absolvierten).

Wir empfinden diese Problematik als außerordentlich spannend und haben deshalb für unser aktuelles Schwerpunktheft wieder Themen ausgewählt, die im Grenzgebiet beider Fächer liegen. In diesem Jahr liegt der Fokus vor allem auf psychiatrischen Symptomen bei neurologischen Erkrankungen. Hierbei haben wir häufige Krankheiten, aber auch häufige Symptome ausgewählt.

In beiden Fächern gibt es darüber hinaus viele Krankheiten und Symptome, auf die beide Fachgebiete einen Anspruch erheben, wie z.B. die Demenz. Diese Konkurrenz sollte konstruktiv genutzt werden. Ideal wäre es, wenn in Klinik und Praxis beide Fachgebiete miteinander verzahnt arbeiten würden, oder als pragmatische Lösung, wenn der Facharzt auch etwas vom anderen Fach versteht. Vielleicht tragen wir mit unserem aktuellen Heft dazu ein wenig bei.

Ich wünsche Ihnen viel Spaß bei der Lektüre!

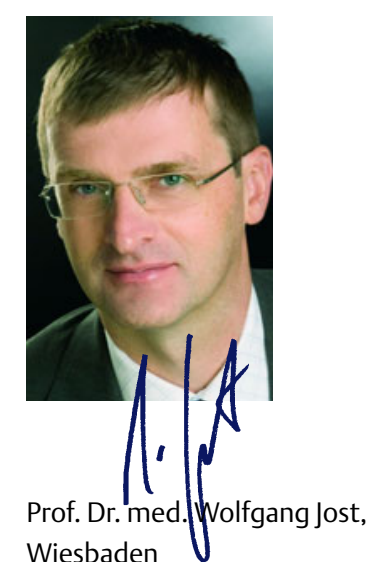

